MS18-P11

\section{Studying weak interactions in crystals at high pressures: when hardware matters}

Marcus Mueller ${ }^{1}$, Boris A. Zakharov ${ }^{2}$, Zoltan Gal ${ }^{3}$, Dyanne

Cruickshank $^{3}$, Elena V. Boldyreva ${ }^{3}$

1. DECTRIS Ltd, Baden-Daettwil, Switzerland

2. Institute of Solid State Chemistry and Mechanochemistry, Siberian Branch of the Russian Academy of Sciences, Novosibirsk, Russia

3. Rigaku Oxford Diffraction, Chalgrove, United Kingdom

email: marcus.mueller@dectris.com

Obtaining reliable information on intermolecular interactions in crystal structures relies on highest-quality diffraction data. This is even more the case when collecting high-pressure data from a sample in a diamond anvil cell (DAC) that limits completeness and observable reciprocal space. For such challenges, hardware becomes a crucial factor. A new generation of laboratory diffractometers using advanced sources and Hybrid Photon Counting (HPC) detectors has been developed, enabling the collection of data at high pressures from even small and weakly diffracting crystals. However, high-quality data alone does not guarantee a high-quality structural model and careful processing is critical for data collected from a sample in a DAC.

We will present a comparison of the results obtained with a previous generation laboratory diffractometer and the latest generation, using a PhotonJet-S microfocus source with silver radiation and a PILATUS3 R CdTe 300K HPC detector. Our results show that the latest generation hardware allows for substantially shorter measurement times, higher data quality, and refinement results not possible with the previous generation. Furthermore, we will discuss the impact of different data processing strategies on the results of crystal structure refinement.

\section{MS18-P12}

\section{Role of size and electronegativity of the transition metal ion, on the high pressure behavior of dimethyl ammonium metal formates}

Abhishek Chitnis ${ }^{1}$, Mirosław Maczka ${ }^{2}$, Himal Bhatt ${ }^{1}$, Mukund Deo ${ }^{1}$, Nandini Garg

1. Bhabha Atomic Research Centre, Mumbai, India

2. Institute of low temperature and structure research, Polish academy of Sciences, Poland, Wrocław, Poland

\section{email: abhishekchitnis87@gmail.com}

Dimethyl ammonium metal formates (DmAMF) are important metal organic framework compounds (MOF). Though, they have been extensively studied at low temperature, to perceive their ferroelectric transition due to H-bonding [1], thermal expansion properties, stress-strain dependence, magneto-electric tunability [2] etc., very few studies have been carried out on these materials at high pressure. As per our studies, application of pressure deforms the formate network. This results in the distortion of the cubo-octahedral cavities formed by it, leading to the structural phase transition [3]. However, the role, of size and electronegativity of the transition metal ion, on this phase transition, has not been addressed so far.

We have studied the dependency of divalent metal ion on pressure evolution of DmAMF (M = Ni, Co, Mn, Cd). Our IR spectroscopic studies show that the framework structure of the formates with transition metal cations having smaller ionic radii $\left[\mathrm{Ni}^{2+}\left(0.69 \mathrm{~A}^{\circ}\right)<\mathrm{Co}^{2+}\left(0.745 \mathrm{~A}^{\circ}\right)<\mathrm{Mn}^{2+}\left(0.83 \mathrm{~A}^{\circ}\right)\right.$ $\left.<\mathrm{Cd}^{2+}\left(0.95 \mathrm{~A}^{\circ}\right)\right]$ distort at higher pressures and the degree of distortion is less than the formates with higher cationic radii. It was also observed that the lower flexibility of the formate network in these compounds can be attributed to the stronger metal-formate coordination bond. In addition, on application of pressure the CNC mode softens [Fig (c) \& (d)] and some of the hydrogen bonds weaken [Fig (a) \& (b)] in the formates having smaller ionic radii cations, indicating that the electronegativity of the metal ion plays a vital role in the high pressure behavior of the coordination and hydrogen bonds. This information can help us to design new MOF's with tailored properties. Further results will be discussed.

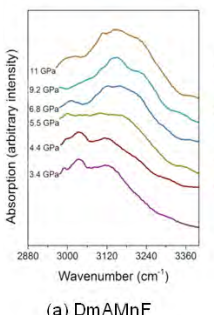

(a) $\mathrm{DmAMnF}$

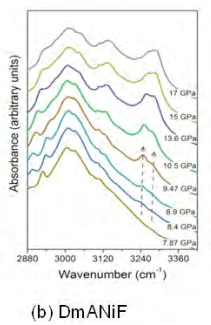

(b) DmANiF

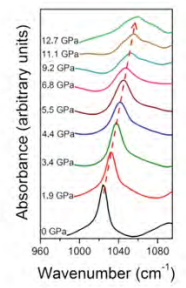

(c) DmAMnF

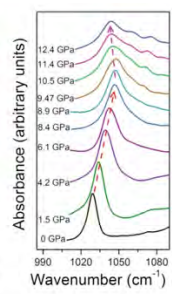

(d) DmANiF
References:

[1] M. Mączka, A. Gągor, B. Macalik, A. Pikul, M. Ptak, and J. Hanuza, Inorg. Chem. 53, 457, 2014.

[2] W. Wang, L. Q. Yan, J. Z. Cong, Y. L. Zhao, F. Wang, S. -P. Shen, T. Zou, D. Zhang, S.G. Wang, X. F. Han \& Y. Sun, Scie. Rep. 3, 2024, 2013

[3] Abhishek Chitnis, H. Bhatt, M. Maczka, M. N. Deo, Nandini Garg, (submitted to Inorganic Chemistry on 15 Feb 2018)

Keywords: MOF, High pressure, IR 